\title{
Semper aliquid haeret: A Paradoxical Embolus lodged in patent foramen ovale
}

\author{
Paula, Pia ; Thali, Michael J ; Ross, Steffen
}

\begin{abstract}
A case of a 54 year old man with unknown medical history suddenly collapsing with cardiac arrest. Post mortem MRI (PMMR) revealed T1 hyperintense material in the pulmonary trunk, a soft tissue edema of the right lower limb, a T2-hyperintense area in the myocardial septum and a patent foramen ovale (PFO). These imaging findings were confirmed by autopsy, which additionally showed a deep vein thrombosis with consecutive central pulmonary embolism and subacute myocardial ischemia. Autopsy showed also a thrombosis of the of the Left Anterior Descending Artery (LAD). PMMR was able to show thrombotic material lodged in the PFO in situ, which was not depictable in autopsy. The combination of the findings of PMMR and autopsy revealed a massive central pulmonary embolism with a paradoxical embolism through a PFO and consecutive coronary thrombosis.
\end{abstract}

DOI: https://doi.org/10.1016/j.jofri.2013.12.003

Posted at the Zurich Open Repository and Archive, University of Zurich

ZORA URL: https://doi.org/10.5167/uzh-87931

Journal Article

Accepted Version

Originally published at:

Paula, Pia; Thali, Michael J; Ross, Steffen (2014). Semper aliquid haeret: A Paradoxical Embolus lodged in patent foramen ovale. Journal of Forensic Radiology and Imaging, 2(1):25-27.

DOI: https://doi.org/10.1016/j.jofri.2013.12.003 


\section{Semper aliquid haeret: A Paradoxical Embolus lodged in patent foramen ovale.}

Authors:

1. P. Paula Dr. med., Institute of Forensic Medicine, University of Zurich, Zurich, Switzerland,

2. M.J. Thali Dr. med., Institute of Forensic Medicine, University of Zurich, Zurich, Switzerland

3. S. Ross Dr. med., Institute of Forensic Medicine, University of Zurich, Zurich, Switzerland

Phone: +41786824995

E-mail: piapaula@gmx.at

Paradoxical Embolism - Case Report

\section{ABSTRACT}

A case of a 54 year old man with unknown medical history suddenly collapsing with cardiac arrest. Post mortem MRI (PMMR) revealed T1 hyperintense material in the pulmonary trunk, a soft tissue edema of the right lower limb, a T2-hyperintense area in the myocardial septum and a patent foramen ovale (PFO). These imaging findings were confirmed by autopsy, which additionally showed a deep vein thrombosis with consecutive central pulmonary embolism and subacute myocardial ischemia. Autopsy showed also a thrombosis of the of the Left Anterior Descending Artery (LAD). PMMR was able to show thrombotic material lodged in the PFO in situ, which was not depictable in autopsy. The combination of the findings of PMMR and autopsy revealed a massive central pulmonary embolism with a paradoxical embolism through a PFO and consecutive coronary thrombosis.

Introduction

Paradoxical Embolism (PDE) can be defined as embolic material, or even air [1] crossing the barrier from the vascular venous to the arterial system via any communication between these two, thus causing clotting within the arterial system. The fetal, physiologically apert foramen ovale closes within the first 2 years of life, with the flap of the foramen ovale (septum primum) closing against the atrial septum (septum secundum), with incomplete fusion in about $25 \%$ of people, consequently resulting in defect. A patent foramen ovale (PFO) is usually an incidental find in a certain 
percentage $(10-30 \%)[2,3]$ of all performed autopsies, usually with no significance, this due to the fact that a PFO is usually somewhat obliterated due to the physiological left-right shunt, as caused by the high left-sided pressure. Any transient increase of the right-sided pressure, as seen in the obstruction of the pulmonary arteries due to pulmonary embolism or severe pulmonary hypertension can, however, lay grounds for the passing of thrombotic material from the venous to the arterial system.

It has been suggested that full closure of the PFO can even occur in adulthood [4] which might explain the coinciding decrease in incidence with increasing age [5].

Case Summary / Case History

We describe the case of a 54 year old innkeeper with a sudden episode of cardiac arrest and no known prior medical history. After spending the night at a friend's apartment, he suddenly collapsed. Upon arrival of the ambulance the patient presented with dilated, unresponsive pupils and asytolia. After approximately 15 minutes of unsuccessful cardiopulmonary resuscitation, death was pronounced. Though a non-criminal death was assumed, a medico-legal autopsy was ordered. The medical history remained unknown as no direct relatives or a treating physician could be detected.

Material \& Methods

Prior to autopsy, a whole body MRI a as well as a Cardiac MRI were performed on a 3T MR scanner (Achieva 3.0 TX, Philips Medical Systems, Best, The Netherlands) using a 16 channel SENSE torso XL coil. The sequences relevant to this study were: axial turbo spin echo (TSE) T2weighted (T2W) sequence with a repetition time (TR) of $8220 \mathrm{~ms}$, echo time (TE) of $60 \mathrm{~ms}$ and a slice thickness of $3.0 \mathrm{~mm}$, and a axial TSE T1-weighted (T1W) sequence with a TR of $694 \mathrm{~ms}$, TE of $6.85 \mathrm{~ms}$, and a slice thickness of $3.0 \mathrm{~mm}$. Whole body PMMR was executed in $5 \mathrm{~mm}$ slice thickness with the q-body coil as coronal T1 (TE 2.3ms, TR 255ms) and coronal inversion recovery (TIRM, TE 70ms, TI 200ms, TR 10300ms).

PMMR \& Autopsy findings 
Post mortem MRI (PMMR) revealed T1-hyperintense material in the pulmonary trunk and a soft tissue edema of the right lower limb, interpreted as indirect signs of a deep vein thrombosis and central pulmonary embolism (Figure 1). Autopsy confirmed this suspicion by showing a deep vein thrombosis (DVT) located in the right lower leg and a central pulmonary thromboembolism. Post mortem cardiac MR showed T2-hyperintense signal alterations in the inferior septal area and the posterior wall of the left ventricle (Figure 2) that gave rise to the suspicion of a subacute ischemic lesion located in this area. Furthermore, cardiac examination during autopsy showed a cardiomegaly (heart weight $500 \mathrm{~g}$ ). A somewhat pale discoloration of the myocardium surrounded by a diffuse red zone was macroscopically identified in the radiologically assumed ischemic area. In addition, a fresh thrombus/embolism, fully obliterating the entire lumen was located in the proximal segment of the Left Anterior Descending Artery (LAD). The right coronary as well as the circumflex coronary artery showed in autopsy non haemodynamic relevant coronary stenoses (up to $50 \%$ ) made up by non-calcified plaques. These two autopsy findings could not be confirmed by PMMR. Additionally, the heart presented in PMMR as well as in autopsy the typical configuration of a patent foramen ovale (PFO) with a septum primum and septum secundum (Figure 3). Furthermore, cardiac MRI revealed a clot-suspicious hyperintensity upon the PFO ("impending paradoxic embolism") (Figure 3b). However this clot was not detected at the autopsy. The lungs showed in PMMR as in autopsy no signs of a pulmonary infarction.

PMMR additionally revealed a small lesion in the brainstem, possibly an infarction scar. However this finding was not found during autopsy.

Histology showed myocardial fibrosis, fragmentation of myocardial fibres but no typical signs of any acute ischemic event. The remaining organs showed age- related alterations. Neither the aorta nor any large vessels showed atherosclerotic lesions. Ultimately the cause of death was heart failure caused by the massive pulmonary thromboembolic event.

Discussion

In the presented case all of the criteria needed to fit the definition of Paradox embolism were 
fulfilled.

Johnson [6] postulated that the diagnostic certainty of a paradoxical embolism can only be achieved when the embolus is actually found adhered to or within the anomalous opening, either by means of radiology or during autopsy. As previously pointed out in the literature, a presumptive diagnosis may be based on simultaneous occurrence of the triad

1) venous thrombosis with or without pulmonary embolism,

2) an intracardiac defect allowing a right to left shunt, and

3) arterial embolism without a corresponding source in the left heart. [7] Although a larger percentage of reported PDEs have been associated with a PFO, there have been reported cases of PDE crossing through a pulmonary arteriovenous malformations, atrial and ventricular defects [8] or even in Ebstein's anomaly [9].

The direct detection of a paradoxical emboli after death has in the past been limited to autopsy. A point of argumentation undoubtedly is whether all cases of an impending embolus on the PFO are actually detected. In the presented case, the lodged embolus was not detected during autopsy. Due to its noninvasive nature, PMMR is a viable tool to depict pathologies which are easily dislodged during the preparation in autopsy (like the thrombembolic material near the PFO). Furthermore, PMMR is an excellent prerequisite to display cardiac pathologies like atrial / septal defects or myocardial infarctions [10].

Reference

1. Lee HY, Yoo SM. A case of paradoxical air embolism in the coronary artery through a patent foramen ovale demonstrated by coronary CT angiography.Clin Imaging. 2013 Jan;37(1):167-9.

2. Penter $P$ (1994) patent foramen ovale: an anatomical study. Apropos of 500 consecutive autopsies. Arch Mal Coeur Vaiss 87:15-21.

3. Kerut et al. (2001) Patent Foramen Ovale: Size and Clinical Significance September. JACC Vol. 
38, No. 3, 2001:613-23.

4. Hagen PT, Scholz DG, Edwards WD. Incidence and size of patent foramen ovale during the first 10 decades of life: an autopsy study of 965 normal hearts. Mayo Clin Proc 1984;59:17-20.

5. Hidehiko Hara, Renu Virmani, Elena Ladich, Shannon Mackey-Bojack, Jack Titus, Mark

Reisman , William Gray , Masato Nakamura, Michael Mooney , Anil Poulose , Robert S. Schwartz.

Patent Foramen ovale: Current Pathology,

Pathophysiology, and Clinical Status Journal of the American College of Cardiology, Volume 46, Issue 9, 1 November 2005, Pages 1768-1776.

6. Johnson B (1951) Paradoxical embolism. J Clin Pathol 4:316

7. Haghi, D. · Sueselbeck, T. · Papavassiliu, T. · Haase, K. · Borggrefe, M., Paradoxical coronary embolism causing non-ST segment elevation myocardial infarction in a case of pulmonary embolism. Z Kardiol. 2004 Oct;93(10):824-8.

8. Loscalzo J, Wachsman DE, Jacobs AK (1986) Paradoxical embolism: clinical presentation, diagnostic strategies, and therapeutic options. Am Heart J 112(1):141- 145

9. Uyan C, Yazici M, Uyan AP, Dokumaci B. Paradoxical embolism in Ebstein's anomaly. Exp Clin Cardiol. 2001 Fall;6(3):173-5.

10. Jackowski C, Christe A, Sonnenschein M, Aghayev E, Thali MJ. Postmortem unenhanced magnetic resonance imaging of myocardial infarction in correlation to histological infarction age characterization. Eur Heart J 2006; 27:2459-2467 
Figure Legends Figure 1 (a) axial T1 PMMR at the level of the pulmonary trunk; (b) coronal STIR PMMR of the lower limb.

a) T1-hyperintense signal alterations in the left pulmonary artery (arrow).

b) DiffuseTIRM-hyperintensesignalalterationssuspiciousofasofttissueedema of the right lower limb.

Figure 2 (a) short axis T2 weighted cardiac PMMR; (b) Autopsy specimen

T2-hyperintense signal alterations in the inferior septal area and the posterior wall of the left ventricle (arrow). Autopsy specimen with a corresponding pale discoloration of the myocardium surrounded by a diffuse red zone.

Figure 3 (a \& b) axial T2 weighted PMMR; (c) Autopsy specimen

a) Foramen ovale with typical configuration of septum primum and septum secundum (arrows). Note the presence of suspected thrombembolic material directly adjacent to the foramen ovale.

b) Open connection between both atriae (arrow) 

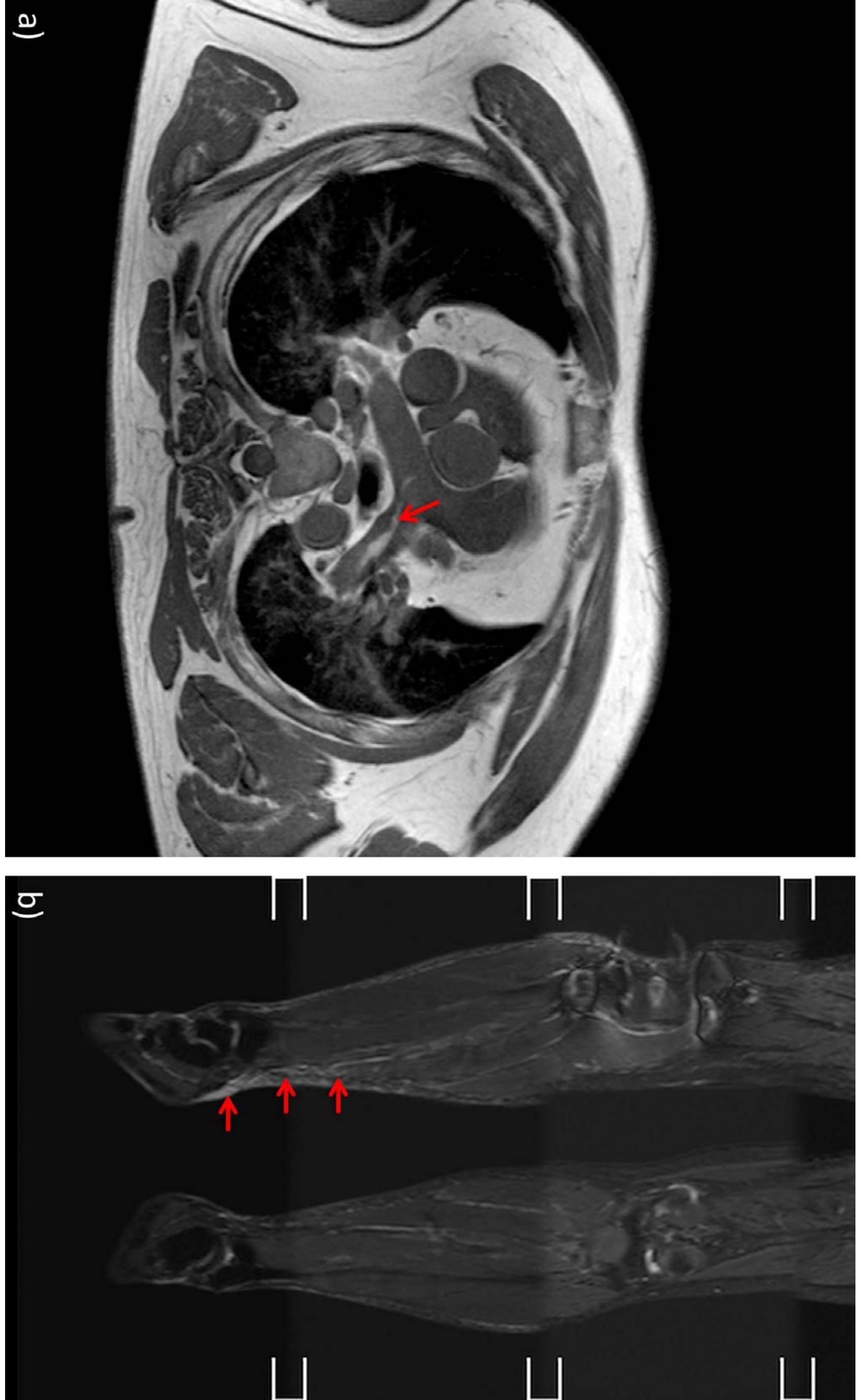

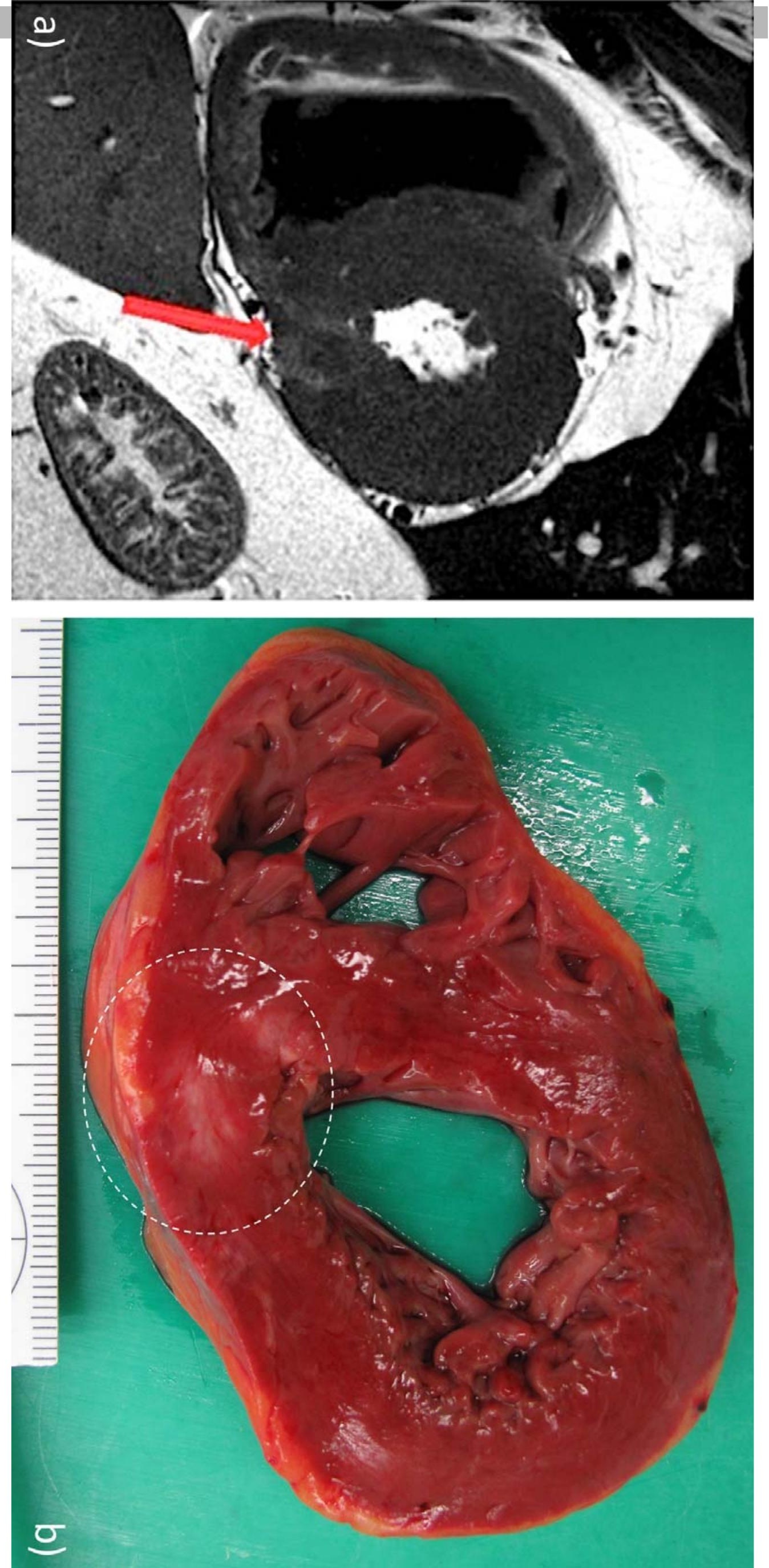

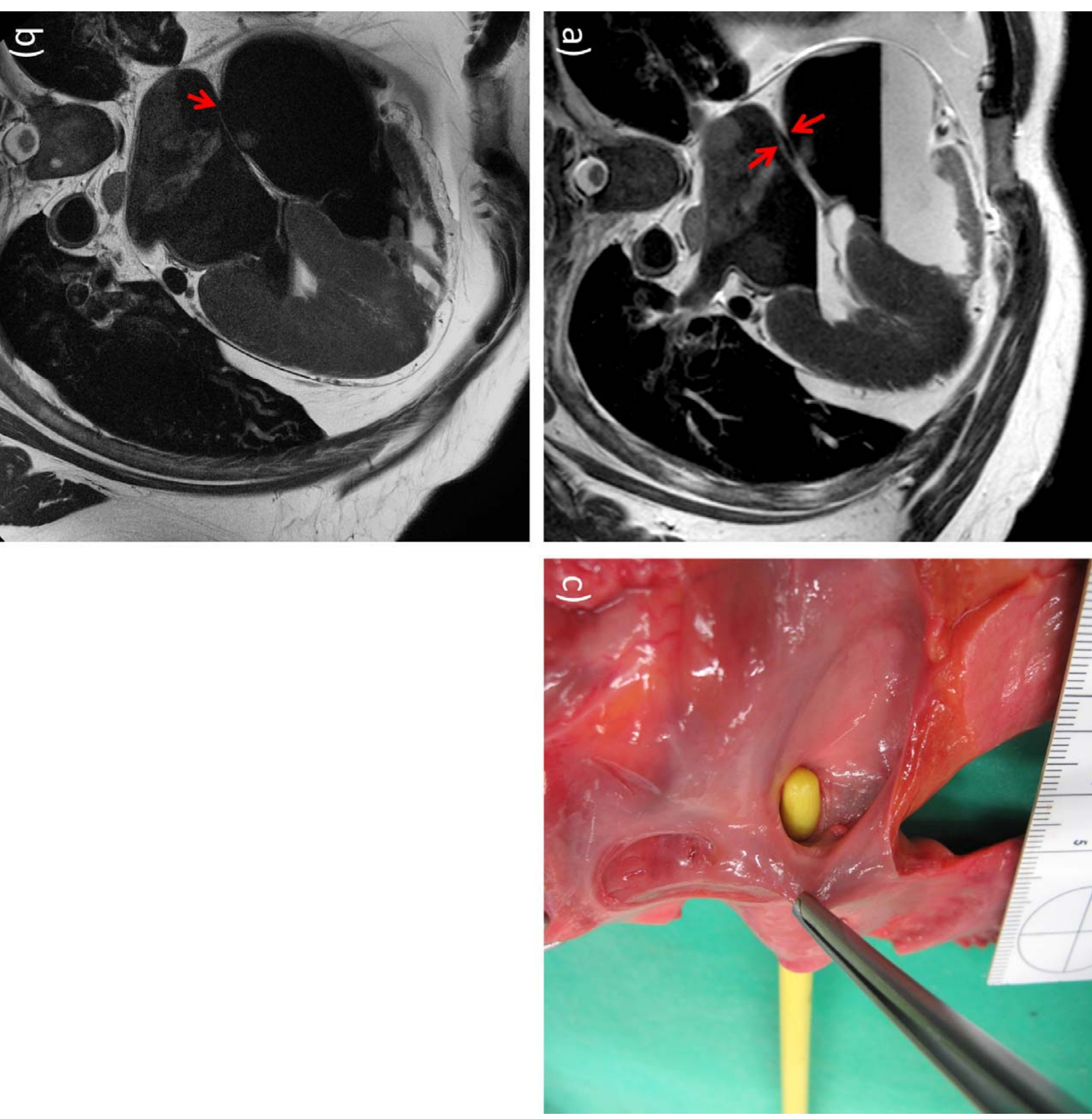